\title{
Evidence for host-dependent RNA editing in the transcriptome of SARS-CoV-2 in humans
}

\author{
Salvatore Di Giorgio ${ }^{1,2}+$, Filippo Martignano ${ }^{1,2}+$, Maria Gabriella Torcia ${ }^{3}$, Giorgio Mattiuz ${ }^{1,3}{ }^{*}$, , Silvestro G. \\ Conticello ${ }^{1,4}+*$
}

${ }^{1}$ Core Research Laboratory, ISPRO, Firenze, 50139, Italy. ${ }^{2}$ Department of Medical Biotechnologies, University of Siena, Siena, 53100, Italy. ${ }^{3}$ Department of Experimental and Clinical Medicine, University of Florence, Firenze 50139, Italy ${ }^{4}$ Institute of Clinical Physiology, National Research Council, 56124, Pisa, Italy.

$\dagger \ddagger$ These authors contributed equally

${ }^{*}$ Corresponding author. Email: giorgio.mattiuz@unifi.it, s.conticello@ispro.toscana.it

The COVID-19 outbreak has become a global health risk and understanding the response of the host to the SARS-CoV-2 virus will help to contrast the disease. Editing by host deaminases is an innate restriction process to counter viruses, and it is not yet known whether it operates against Coronaviruses. Here we analyze RNA sequences from bronchoalveolar lavage fluids derived from infected patients. We identify nucleotide changes that may be signatures of RNA editing: Adenosine-to-Inosine changes from ADAR deaminases and Cytosine-to-Uracil changes from APOBEC ones. A mutational analysis of genomes from different strains of human-hosted Coronaviridae reveals mutational patterns compatible to those observed in the transcriptomic data. Our results thus suggest that both APOBECs and ADARs are involved in Coronavirus genome editing, a process that may shape the fate of both virus and patient.

\section{Introduction}

Emerging viral infections represent a threat to global health, and the recent outbreak of Novel Coronavirus Disease 2019 (COVID-19) caused by Severe Acute Respiratory Syndrome Coronavirus 2 (SARS-CoV-2, Novel Coronavirus, 2019-nCoV) exemplifies the risks $(1,2)$. As viruses are obligate intracellular parasites, organisms have evolved innate immune processes to sense and counter the viruses. Among these, RNA and DNA editing mediated by endogenous deaminases can provide a potent restriction against specific viruses. Two deaminase families are present in mammalian species: the ADARs target double stranded RNA (dsRNA) for deamination of Adenines into Inosines (A-to-I) $(3,4)$, and the APOBECs deaminate Cytosines into Uracils (C-to-U) on single-stranded nucleic acids (ssDNA and ssRNA) $(5,6)$. During viral infections ADARs act either directly, through hypermutation of the viral RNA, or indirectly, through editing of host transcripts that modulate the cellular response (7-18). On the other hand, APOBECs target the viral genome, typically DNA intermediates (19-26), either through C-to-U hypermutation or through a non-enzymatic path that interferes with reverse transcription $(27,28)$. Some APOBEC3 proteins can interfere in vitro with Coronaviridae replication, yet it is not clear whether their enzymatic activity is involved (29). Eventually though, these restriction systems can also be exploited by the viruses to support their infectivity and increase their evolutionary potential $(9,11-15$, 30-32).

First release: 18 May 2020

\section{Results}

To assess whether RNA editing could be involved in the response to SARS-CoV-2 infections, we started from publicly available RNA sequencing datasets from bronchoalveolar lavage fluids (BALF) obtained from patients diagnosed with COVID-19. While transcriptomic data for all samples could be aligned to the SARS-CoV-2 reference genome, the quality of the sequencing varied and only eight samples had coverage and error rates suitable for the identification of potentially edited sites (Data S1). We called single nucleotide variants (SNVs) on these eight samples $(33,34)$ using REDItools $2(35-$ 37) and JACUSA (38) using the following thresholds: reads supporting the $\mathrm{SNV} \geq 4$, allelic fraction $\geq 0.5 \%$, coverage $\geq 20$, quality of the reads $>25$, base quality $>35$ (Fig. S1A). The two pipelines gave comparable results with $\sim 50 \%$ of the SNV positions called by both (Fig. S1B, Fig S2). We identified 910 SNVs common to REDItools 2 and JACUSA, ranging from 24 to 238 SNVs per sample (Fig. 1, Data S3). Given the thresholds used to call the SNV, samples with lower sequencing depths displayed lower numbers of SNVs.

While the weight of each SNV type varies across samples (Fig. 1), a bias toward transitions is always present, which is even more evident when all mutational data are pooled (Fig. 2A, B). This holds true even when only SNVs recurring in more samples are considered (Fig. 2C).

SNV frequency and number of transversions are compatible with mutation rates observed in Coronaviruses $\left(10^{-6 /-7}\right.$, (39) and commonly associated to the RNA dependent RNA polymerases (RdRp). RdRp are error-prone and are 
considered the main source of mutations in RNA viruses. However, the Coronavirus nsp14-ExoN gene provides a form of error correction (40), which is probably the reason mutation rates in Coronaviruses are lower than those observed in RNA viruses with smaller genomes. The mutational spectrum in SARS quasispecies presents a very weak bias toward U-toG. Inactivation of nsp14-ExoN error correction reveals the mutational spectrum of the RdRp, which is quite different from the pattern we observe (i.e., main changes are C-to-A, followed by U-to-C, G-to-U, A-to-C and U-to-G) (41). As such we would consider that SNVs deriving from RdRp errors represent a marginal fraction of the SNVs in the SARS-CoV-2 samples.

The bias toward transitions -mainly $A>G / T>C$ changesresembles the pattern of SNVs observed in human transcriptomes $(42)$ or in viruses $(8,10,18)$, where $\mathrm{A}>\mathrm{G}$ changes derive from deamination of A-to-I mediated by the ADARs. It is thus likely that also in the case of SARS-CoV-2 these $A>G / T>C$ changes are due to the action of the ADARs.

$\mathrm{C}>\mathrm{T}$ and $\mathrm{G}>\mathrm{A}$ SNVs are the second main group of changes and could derive from APOBEC-mediated C-to-U deamination. Contrary to A-to-I, C-to-U editing is a relatively rare phenomenon in the human transcriptome (42) and, with regard to viruses, it has been associated only to positive-sense ssRNA Rubella virus (32), where $\mathrm{C}>\mathrm{T}$ changes represent the predominant SNV type. The observation that only A-to-I editing is present in RNA viruses that infect non-vertebrate animals, where RNA-targeting APOBECs are not present $(10,18)$, supports the hypothesis that APOBECs are involved in the RNA editing of this human-targeting virus.

A third group of SNVs, $\mathrm{A}>\mathrm{T} / \mathrm{T}>\mathrm{A}$ transversions, is also present. While this type of SNVs has been described (43), its origin is yet unknown.

$A>G$ and $T>C$ changes are evenly represented for SNV frequency (Fig. 2A), for number of unique SNVs (Fig. 2B, C), and for distribution on the viral genome (Fig. 2D). As ADARs target dsRNA, this suggests that dsRNA encompasses the entire genome. While dsRNA in human transcripts are often driven by inverted repeats, the most likely source of dsRNA in the viral transcripts is the replication, where both positive and negative strands are present and can result in wide regions of double-stranded RNA.

Contrary to A-to-I changes, C-to-U changes are biased toward the positive-sense strand (Fig. 2B, C, D, $p<0.0001$ ). Since ADARs and APOBECs target selectively dsRNA and ssRNA, such distribution could derive from the presence at all times of RNA in a dynamic equilibrium between doublestrandedness -when negative-sense RNA is being transcribedand single-strandedness -when nascent RNA is released. Some areas seem to bear less SNVs, but this might be related to lower sequencing depth in those regions.

As APOBEC deaminases preferentially target cytosines within specific sequence contexts, we analyzed the nucleotide context of A-to-I and C-to-U SNVs in the viral genome (Fig. $3 \mathrm{~A}, \mathrm{~B})$. A slight depletion of $\mathrm{G}$ bases in position -1 is present at A-to-I edited positions. The depletion is not as strong as the signal previously reported in human transcripts (44-47). The low editing frequencies we observe resembles the editing present on human transcripts containing Alu sequences, which were in a limited number in those early datasets. Indeed, there is no evidence of a sequence context preference if we use a larger dataset such as REDIportal (48), which includes $>1.5 \mathrm{M}$ sites in Alu repeats (Fig. S3).

On the other hand, C-to-U changes preferentially occur $3{ }^{\prime}$ to thymines and adenines, a sequence context that resembles the one observed for APOBEC1-mediated deamination ([AU]C[AU]) $(49,50)$.

We then aligned available genomes from SARS-CoV-2, Middle East Respiratory Syndrome-related Coronavirus (MERS-CoV), and the Severe Acute Respiratory Syndrome Coronavirus (SARS-CoV) to test whether RNA editing could be responsible for some of the mutations acquired through evolution. Indeed, the genomic alignments reveal that a substantial fraction of the mutations in all strains could derive from enzymatic deamination (Fig. 4A, B, C), with a prevalence of C-to-U mutations, and a sequence context compatible with APOBEC-mediated editing exists also in the genomic $\mathrm{C}$ to-U SNVs (Fig. 4D, E, F).

\section{Discussion}

Considering the source of our analysis -metagenomic sequencing-, we wonder whether the low-level editing we observe $(\sim 1 \%)$ reflects the levels of editing of the viral transcripts inside the cells. Indeed, beside a small fraction of cellular transcripts edited at high-frequency, most ADARedited sites in the human transcriptome (typically inside Alu sequences) present editing levels of $\sim 1 \%(4,42,51)$. It has been shown that a fraction of the cellular transcripts is hyperedited by ADARs (52-54). While we were unable to observe hyperedited reads in the metagenomic samples, it is possible that hyperedited transcripts fail to be packaged into the virus.

With regard to APOBEC-mediated RNA editing, its detection in the viral transcriptomes is already indicative, as this type of editing is almost undetectable in human tissues (42). Such enrichment points either toward an induction of the APOBECs triggered by the infection, or to specific targeting of the APOBECs onto the viral transcripts. The APOBECs have been proved effective against many viral species in experimental conditions, yet, until now their mutational activity in clinical settings has been shown only in a handful of viral infections (19-26) through DNA editing- and in Rubella virus, on RNA (32).

As in Rubella virus, we observe a bias in APOBEC editing 
toward the positive-sense strand. This bias and the low editing frequencies might be indicative of the dynamics of the virus, from transcription to selection of viable genomes. It is reasonable to assume that sites edited on the negative-sense strand will result in a mid-level editing frequency, as not all negative-sense transcripts will be edited (Fig. 5A). On the other hand, editing of the positive-sense strand can occur upon entry of the viral genome, thus yielding high-frequency editing (Fig. 5B), or after viral genome replication, resulting in low-frequency editing (Fig. 5C). Indeed, lack of a sizable fraction of highly edited $\mathrm{C}>\mathrm{T}$ SNVs suggests that APOBEC editing occurs late in the viral lifecycle (Fig. 5C). Yet, since they occur earlier, G>A SNVs should be closer in number to $\mathrm{C}>\mathrm{T}$ ones and with higher levels of editing, which is not what we observe (Fig. 2A, B, C). The overrepresentation of C>T SNVs could be due to an imbalance toward positive-sense transcripts, as these are continuously generated from the negative-sense ones (and double-stranded hybrid RNAs are lost). However, the editing frequencies of $\mathrm{G}>\mathrm{A}$ SNVs should be much higher as $\mathrm{G}>\mathrm{A}$ SNVs are generated upstream to the $\mathrm{C}>\mathrm{T}$ ones. A more fitting explanation is that editing of the negative-sense transcripts results in loss of the edited transcript (Fig. 5D), possibly because editing triggers nonsense mediated decay (55), thus lowering the chances of the edited site to be transmitted.

Since most of the APOBECs are unable to target RNA, the only well characterized cytidine-targeting deaminase is APOBEC1, mainly expressed in the gastrointestinal tract, and APOBEC3A (56), whose physiological role is not clear. As with A-to-I editing, it will be important to assess the true extent of APOBEC RNA editing in infected cells.

The functional meaning of RNA editing in SARS-CoV-2 is yet to be understood. In other contexts, editing of the viral genome determines its demise or fuels its evolution. For DNA viruses, the selection is indirect, as genomes evolve to reduce potentially harmful editable sites (e.g., (18), but for RNA viruses this pressure is even stronger, as RNA editing directly affects the genetic information and efficiently edited sites disappear.

A comparison of the SNV datasets from the transcriptomic and from the genomic analyses reveals a different weight of A-to-I and C-to-U changes (Fig. 2B, Fig. 4A), with an underrepresentation of A-to-I in the viral genomes. As our analysis underestimates the amount of editing due to strict parameters used, it could be possible that A-to-I SNVs are not fixed in the viral population when ADAR editing is such that impairs the viral genome.

An analysis of the outcomes of the mutations is difficult due to the low numbers of events collected so far, but there are some trends that might be suggestive (Data S2). C-to-U changes leading to Stop codons are overrepresented in the transcriptomic data but -as expected- disappear in the genomic dataset. This might point -again- to an antiviral role for these editing enzymes. There is also an underrepresentation of $\mathrm{C}>\mathrm{T}$ missense mutations, but its meaning is difficult to interpret.

Finally, this analysis is a first step in understanding the involvement of RNA editing in viral replication, and it could lead to clinically relevant outcomes: (a) if these enzymes are relevant in the host response to Coronavirus infection, a deletion polymorphism quite common in the Chinese population, encompassing the end of APOBEC3A and most of APOBEC3B (57, 58) could play a role in the spread of the infection. (b) Since RNA editing and selection act orthogonally in the evolution of the viruses, comparing genomic sites that are edited with those that are mutated could lead to the selection of viral regions potentially exploitable for therapeutic uses.

\section{Materials and Methods \\ Sequencing Data}

RNA sequencing data available from projects PRJNA601736, PRJNA603194 and PRJNA605907 were downloaded from NCBI (https://www.ncbi.nlm.nih.gov/sra/) using the FASTQdump utilities from the SRA-toolkit with the command line: prefetch -v SRR* \&\& fastq-dump --outdir /path_dir/ | --splitfiles /path_dir/SRR*.sra

Since most of the reads of samples from PRJNA605907 were missing their mate, forward-reads and reverse-reads from these samples have been merged in a single FASTQ, which is treated as a single-end experiment. Details of the sequencing runs are summarized in Data S1.

\section{Data pre-processing}

SRR11059940, SRR11059941, SRR11059942 and SRR11059945 showed a reduced quality of the sequencing in the terminal part of the reads.

We used TRIMMOMATIC (59) to trim the reads of those samples to $100 \mathrm{bp}$, with the following command line: trimmomatic SE SRR*.fastq SRR*.trimmed.fastq CROP:100

We aligned the FASTQ files using Burrows-Wheeler Aligner (BWA) (60) using the official sequence of SARS-CoV2 (NC_045512. 2) as reference genome. After the alignments BAM files were sorted them using SAMtools (61).

Command line used for paired-end samples: bwa mem NC_045512.2.fa SRR*_1.fastq SRR*_2.fastq | samtools sort -O BAM -o SRR*_.bam

Command line used for single-end samples: bwa mem NC_045512.2.fa SRR*.fastq | samtools sort -O BAM -o SRR*_.bam

The aligned bams have been analyzed with QUALIMAP (62). Due to a high error rate reported by QUALIMAP, samples SRR11059943 and SRR10971381 have been removed from the analysis. 


\section{Single nucleotide variant (SNV) calling}

A diagram of the entire pipeline is shown in Fig. S1A. We used RediTools $2(35,37)$ and JACUSA $(38)$ to call the SNVs_using the command line:

python 2.7 reditools.py -f SRR* ${ }^{*}$.bam -o

SRR10903401_stat_table_allPos.txt -S -s 0 -os 4 -m

/homol_site/SRR*_homopol.txt -c SRR ${ }^{*}$ _homopol.txt -r

/Reference/NC_045512.2.fa -a SRR*_stat_table_allPos.txt -q

25 -bq 35 -mbp 15 -Mbp 15

jacusa call-1 -p 20 -r SRR*.vcf -a B,I,Y -s -f V -q 35 -m 25

$\mathrm{SRR}^{*}$.srt.bam

With regard to REDItools 2, we removed all SNVs within 15 nucleotides from the beginning or the end of the reads to avoid artifacts due to misalignments.

To avoid potential artifacts due to strand bias, we used the AS_StrandOddsRatio parameter calculated following GATK guidelines (https://gatk.broadinstitute.org/hc/enus/articles/360040507111-AS-StrandOddsRatio), and any mutation with a AS_StrandOddsRatio $>4$ has been removed from the dataset.

Bcftools (61) has been used to calculate total allelic depths on the forward and reverse strand (ADF, ADR) for AS_StrandOddsRatio calculation, with the following command line:

Mpileup -a

FORMAT/AD,FORMAT/ADF,FORMAT/ADR,FORMAT/DP,F

ORMAT/SP -O v -A -C -I -d 1000000 -q 25 -Q 35 -f

NC_045512.2.fa -o SRR*.vcf SRR*.srt.bam

Mutations common to the datasets generated by Reditools 2 and JACUSA were considered $(n=910$, Fig. S2, Data S3). The threshold we used to filter the SNVs is based on minimum coverage (20 reads), number of supporting reads (at least 4 mutated reads), allelic fraction (0.5\%), quality of the mapped reads $(>25)$, and base quality $(>35)$. In the dataset there were only 6 SNVs with allelic fractions in the range 30$85 \%(C>T, 1 ; T>C, 3 ; G>T, 2)$. Since there were no SNVs with higher allelic fractions, we presume that all samples originated from the same viral strain.

Recurring SNVs have been defined as the SNVs present in at least two samples. In order to overcome the problem of samples with lower sequencing depth, we used the positions of the SNVs common to both REDItools 2 and JACUSA to call again the SNVs irrespectively of the number of supporting reads.

\section{Data manipulation}

$\mathrm{R}$ packages (Biostrings, rsamtools, ggseqlogo ggplot2, splitstackshape) and custom Perl scripts were used to handle the data.

\section{Sequence context analysis}

Logo alignments were calculated using ggseqlogo, using either the pooled dataset of the dataset of recurring SNVs. Logo alignments the human edited sites were performed using ADAR sites from REDIportal (48) that were shared by at least 4 samples. SARS-CoV-2, SARS and MERS genomic data were prepared for the Logi alignment using the GenomicRanges R package (63).

\section{SNV calling in genomic data from SARS-CoV-2, SARS and MERS}

The viral genomic sequences of MERS (taxid:1335626) and SARS (taxid:694009) were selected on NCBI Virus (https://www.ncbi.nlm.nih.gov/labs/virus/vssi/\#/) using the query: Host: Homo Sapiens (human), taxid:9606; -Nucleotide Sequence Type: Complete. They were aligned using the "Align" utility. Consensus sequences of SARS and MERS genomes were built using the "cons" tool from the EMBOSS suite (http://bioinfo.nhri.org.tw/gui/) with default settings.

SARS-CoV-2 genomic sequences were downloaded from GISAID (https://www.gisaid.org/) and aligned with MUSCLE (64).

SNVs have been called with a custom R script, by comparing viral genome sequences to the respective consensus sequence or, for SARS-CoV-2, to the NC_045512.2 reference sequence. SNVs, viral consensus sequences, and Coronoviradae genome sequences identifiers, are provided in Data S3, S4 and S5.

\section{SNVs annotation}

SNVs (from both genomic and somatic SNVs sets) occurring on coding sequences have been annotated with custom $\mathrm{R}$ scripts to determine the outcome of the nucleotide change (nonsense/missense/synonymous mutation). A summary is reported in Data S2.

\section{Statistical Analysis}

fisher.test() function from the $\mathrm{R}$ base package has been used for all the statistical tests. To test the significance of C-to- $\mathrm{U}$ bias on the positive strand, we compared $\mathrm{C}>\mathrm{T} / \mathrm{G}>\mathrm{A}$ SNV counts to the count of $\mathrm{C} / \mathrm{G}$ bases on the reference genome. For P-values of "RNA vs Reference", "DNA vs Reference", and "genome vs RNA" $2 \times 2$ contingency tables have been generated as shown in Data S2.

\section{REFERENCES AND NOTES}

1. F. Wu, S. Zhao, B. Yu, Y.-M. Chen, W. Wang, Z.-G. Song, Y. Hu, Z.-W. Tao, J.-H. Tian, Y.-Y. Pei, M.-L. Yuan, Y.-L. Zhang, F.-H. Dai, Y. Liu, Q.-M. Wang, J.-J. Zheng, L. Xu, E. C. Holmes, Y.-Z. Zhang, A new coronavirus associated with human respiratory disease in China. Nature 579, 265-269 (2020). doi:10.1038/s41586-020-20083 Medline

2. Q. Li, X. Guan, P. Wu, X. Wang, L. Zhou, Y. Tong, R. Ren, K. S. M. Leung, E. H. Y. Lau, J. Y. Wong, X. Xing, N. Xiang, Y. Wu, C. Li, Q. Chen, D. Li, T. Liu, J. Zhao, M. Liu, W. Tu, C. Chen, L. Jin, R. Yang, Q. Wang, S. Zhou, R. Wang, H. Liu, Y. Luo, Y. Liu, G. Shao, H. Li, Z. Tao, Y. Yang, Z. Deng, B. Liu, Z. Ma, Y. Zhang, G. Shi, T. T. Y. Lam, J. T. Wu, G. F. Gao, B. J. Cowling, B. Yang, G. M. Leung, Z. Feng, Early 
Transmission Dynamics in Wuhan, China, of Novel Coronavirus-Infected Pneumonia. N. Engl. J. Med. 382, 1199-1207 (2020). doi:10.1056/NEJMoa2001316 Medline

3. M. A. O'Connell, N. M. Mannion, L. P. Keegan, The Epitranscriptome and Innate Immunity. PLOS Genet. 11, e1005687 (2015). doi:10.1371/journal.pgen.1005687 Medline

4. E. Eisenberg, E. Y. Levanon, A-to-I RNA editing - immune protector and transcriptome diversifier. Nat. Rev. Genet. 19, 473-490 (2018) doi:10.1038/s41576-018-0006-1 Medline

5. R. S. Harris, J. P. Dudley, APOBECs and virus restriction. Virology 479-480, 131145 (2015). doi:10.1016/j.virol.2015.03.012 Medline

6. J. D. Salter, H. C. Smith, Modeling the Embrace of a Mutator: APOBEC Selection of Nucleic Acid Ligands. Trends Biochem. Sci. 43, 606-622 (2018). doi:10.1016/i.tibs.2018.04.013 Medline

7. D. R. Taylor, M. Puig, M. E. R. Darnell, K. Mihalik, S. M. Feinstone, New antiviral pathway that mediates hepatitis $C$ virus replicon interferon sensitivity through ADAR1. J. Virol. 79, 6291-6298 (2005). doi:10.1128/JVl.79.10.6291-6298.2005 Medline

8. R. C. Zahn, I. Schelp, O. Utermöhlen, D. von Laer, A-to-G hypermutation in the genome of lymphocytic choriomeningitis virus. J. Virol. 81, 457-464 (2007). doi:10.1128/JVI.00067-06 Medline

9. A. Phuphuakrat, R. Kraiwong, C. Boonarkart, D. Lauhakirti, T.-H. Lee, P. Auewarakul, Double-stranded RNA adenosine deaminases enhance expression of human immunodeficiency virus type 1 proteins. J. Virol. 82, 10864-10872 (2008). doi:10.1128/JVI.00238-08 Medline

10. J. A. Carpenter, L. P. Keegan, L. Wilfert, M. A. O'Connell, F. M. Jiggins, Evidence for ADAR-induced hypermutation of the Drosophila sigma virus (Rhabdoviridae). BMC Genet. 10, 75 (2009). doi:10.1186/1471-2156-10-75 Medline

11. M. Doria, F. Neri, A. Gallo, M. G. Farace, A. Michienzi, Editing of HIV-1 RNA by the double-stranded RNA deaminase ADAR1 stimulates viral infection. Nucleic Acids Res. 37, 5848-5858 (2009). doi:10.1093/nar/gkp604 Medline

12. C. X. George, Z. Li, K. M. Okonski, A. M. Toth, Y. Wang, C. E. Samuel, Tipping the balance: Antagonism of PKR kinase and ADAR1 deaminase functions by virus gene products. J. Interferon Cytokine Res. 29, 477-487 (2009). doi:10.1089/iir.2009.0065 Medline

13. G. Clerzius, J.-F. Gélinas, A. Gatignol, Multiple levels of PKR inhibition during HIV1 replication. Rev. Med. Virol. 21, 42-53 (2010). doi:10.1002/rmv.674 Medline

14. M. Doria, S. Tomaselli, F. Neri, S. A. Ciafrè, M. G. Farace, A. Michienzi, A. Gallo, ADAR2 editing enzyme is a novel human immunodeficiency virus-1 proviral factor. J. Gen. Virol. 92, 1228-1232 (2011). doi:10.1099/vir.0.028043-0 Medline

15. C. K. Pfaller, Z. Li, C. X. George, C. E. Samuel, Protein kinase PKR and RNA adenosine deaminase ADAR1: New roles for old players as modulators of the interferon response. Curr. Opin. Immunol. 23, 573-582 (2011). doi:10.1016/i.coi.2011.08.009 Medline

16. C. E. Samuel, ADARs: Viruses and innate immunity. Curr. Top. Microbiol. Immunol. 353, 163-195 (2011). doi:10.1007/82 2011148 Medline

17. S. Tomaselli, F. Galeano, F. Locatelli, A. Gallo, ADARs and the Balance Game between Virus Infection and Innate Immune Cell Response. Curr. Issues Mol. Biol. 17, 37-51 (2015). Medline

18. U. Rosani, C.-M. Bai, L. Maso, M. Shapiro, M. Abbadi, S. Domeneghetti, C.-M. Wang, L. Cendron, T. MacCarthy, P. Venier, A-to-I editing of Malacoherpesviridae RNAs supports the antiviral role of ADAR1 in mollusks. BMC Evol. Biol. 19, 149 (2019). doi:10.1186/s12862-019-1472-6 Medline

19. J. P. Vartanian, A. Meyerhans, B. Asjö, S. Wain-Hobson, Selection, recombination, and G----A hypermutation of human immunodeficiency virus type 1 genomes. J.
Virol. 65, 1779-1788 (1991). doi:10.1128/JVI.65.4.1779-1788.1991 Medline

20. R. S. Harris, K. N. Bishop, A. M. Sheehy, H. M. Craig, S. K. Petersen-Mahrt, I. N. Watt, M. S. Neuberger, M. H. Malim, DNA deamination mediates innate immunity to retroviral infection. Cell 113, 803-809 (2003). doi:10.1016/S00928674(03)00423-9 Medline

21. R. Mahieux, R. Suspène, F. Delebecque, M. Henry, O. Schwartz, S. Wain-Hobson, J.-P. Vartanian, Extensive editing of a small fraction of human T-cell leukemia virus type 1 genomes by four APOBEC3 cytidine deaminases. J. Gen. Virol. 86, 2489-2494 (2005). doi:10.1099/vir.0.80973-0 Medline

22. C. Noguchi, H. Ishino, M. Tsuge, Y. Fujimoto, M. Imamura, S. Takahashi, K. Chayama, G to A hypermutation of hepatitis B virus. Hepatology 41, 626-633 (2005). doi:10.1002/hep.20580 Medline

23. J.-P. Vartanian, D. Guétard, M. Henry, S. Wain-Hobson, Evidence for editing of human papillomavirus DNA by APOBEC3 in benign and precancerous lesions. Science 320, 230-233 (2008). doi:10.1126/science.1153201 Medline

24. R. Suspène, M.-M. Aynaud, S. Koch, D. Pasdeloup, M. Labetoulle, B. Gaertner, J.P. Vartanian, A. Meyerhans, S. Wain-Hobson, Genetic editing of herpes simplex virus 1 and Epstein-Barr herpesvirus genomes by human APOBEC3 cytidine deaminases in culture and in vivo. J. Virol. 85, 7594-7602 (2011) doi:10.1128/JVI.00290-11 Medline

25. J. M. Cuevas, R. Geller, R. Garijo, J. López-Aldeguer, R. Sanjuán, Extremely High Mutation Rate of HIV-1 In Vivo. PLOS Biol. 13, e1002251 (2015). doi:10.1371/journal.pbio.1002251 Medline

26. A. Peretti, E. M. Geoghegan, D. V. Pastrana, S. Smola, P. Feld, M. Sauter, S. Lohse, M. Ramesh, E. S. Lim, D. Wang, C. Borgogna, P. C. FitzGerald, V. Bliskovsky, G. J. Starrett, E. K. Law, R. S. Harris, J. K. Killian, J. Zhu, M. Pineda, P. S. Meltzer, R. Boldorini, M. Gariglio, C. B. Buck, Characterization of BK Polyomaviruses from Kidney Transplant Recipients Suggests a Role for APOBEC3 in Driving In-Host Virus Evolution. Cell Host Microbe 23, 628-635.e7 (2018). doi:10.1016/j.chom.2018.04.005 Medline

27. E. N. C. Newman, R. K. Holmes, H. M. Craig, K. C. Klein, J. R. Lingappa, M. H. Malim, A. M. Sheehy, Antiviral function of APOBEC3G can be dissociated from cytidine deaminase activity. Curr. Biol. 15, 166-170 (2005). doi:10.1016/i.cub.2004.12.068 Medline

28. D. Pollpeter, M. Parsons, A. E. Sobala, S. Coxhead, R. D. Lang, A. M. Bruns, S. Papaioannou, J. M. McDonnell, L. Apolonia, J. A. Chowdhury, C. M. Horvath, M. H. Malim, Deep sequencing of HIV-1 reverse transcripts reveals the multifaceted antiviral functions of APOBEC3G. Nat. Microbiol. 3, 220-233 (2018). doi:10.1038/s41564-017-0063-9 Medline

29. A. Milewska, E. Kindler, P. Vkovski, S. Zeglen, M. Ochman, V. Thiel, Z. Rajfur, K. Pyrc, APOBEC3-mediated restriction of RNA virus replication. Sci. Rep. 8, 5960 (2018). doi:10.1038/s41598-018-24448-2 Medline

30. J. S. Albin, G. Haché, J. F. Hultquist, W. L. Brown, R. S. Harris, Long-term restriction by $A P O B E C 3 F$ selects human immunodeficiency virus type 1 variants with restored Vif function. J. Virol. 84, 10209-10219 (2010). doi:10.1128/JVI.00632-10 Medline

31. H. A. Sadler, M. D. Stenglein, R. S. Harris, L. M. Mansky, APOBEC3G contributes to HIV-1 variation through sublethal mutagenesis. J. Virol. 84, 7396-7404 (2010). doi:10.1128/JVI.00056-10 Medline

32. L. Perelygina, M.-H. Chen, S. Suppiah, A. Adebayo, E. Abernathy, M. Dorsey, L. Bercovitch, K. Paris, K. P. White, A. Krol, J. Dhossche, I. Y. Torshin, N. Saini, L. J. Klimczak, D. A. Gordenin, A. Zharkikh, S. Plotkin, K. E. Sullivan, J. Icenogle, Infectious vaccine-derived rubella viruses emerge, persist, and evolve in cutaneous granulomas of children with primary immunodeficiencies. PLOS Pathog. 15, e1008080 (2019). doi:10.1371/journal.ppat.1008080 Medline

33. L. Chen, W. Liu, Q. Zhang, K. Xu, G. Ye, W. Wu, Z. Sun, F. Liu, K. Wu, B. Zhong, Y. 
Mei, W. Zhang, Y. Chen, Y. Li, M. Shi, K. Lan, Y. Liu, RNA based mNGS approach identifies a novel human coronavirus from two individual pneumonia cases in 2019 Wuhan outbreak. Emerg. Microbes Infect. 9, 313-319 (2020). doi:10.1080/22221751.2020.1725399 Medline

34. Z. Shen, Y. Xiao, L. Kang, W. Ma, L. Shi, L. Zhang, Z. Zhou, J. Yang, J. Zhong, D. Yang, L. Guo, G. Zhang, H. Li, Y. Xu, M. Chen, Z. Gao, J. Wang, L. Ren, M. Li, Genomic Diversity of Severe Acute Respiratory Syndrome-Coronavirus 2 in Patients With Coronavirus Disease 2019. Clin. Infect. Dis. ciaa203 (2020). doi:10.1093/cid/ciaa203 Medline

35. E. Picardi, G. Pesole, REDItools: High-throughput RNA editing detection made easy. Bioinformatics 29, 1813-1814 (2013). doi:10.1093/bioinformatics/btt287 Medline

36. C. Lo Giudice, M. A. Tangaro, G. Pesole, E. Picardi, Investigating RNA editing in deep transcriptome datasets with REDItools and REDIportal. Nat. Protoc. 15 , 1098-1131 (2020). doi:10.1038/s41596-019-0279-7 Medline

37. T. Flati, S. Gioiosa, N. Spallanzani, I. Tagliaferri, M. Angela Diroma, G. Pesole, G. Chillemi, E. Picardi, T. Castrignanò, HPC-REDItools: A Novel HPC-aware Tool for Improved Large Scale RNA-editing Analysis. BMC Bioinformatics, doi: $10.1101 / 2020.04 .30 .069732(2020)$

38. M. Piechotta, E. Wyler, U. Ohler, M. Landthaler, C. Dieterich, JACUSA: Sitespecific identification of RNA editing events from replicate sequencing data. BMC Bioinformatics 18, 7 (2017). doi:10.1186/s12859-016-1432-8 Medline

39. L. D. Eckerle, M. M. Becker, R. A. Halpin, K. Li, E. Venter, X. Lu, S. Scherbakova, R. L. Graham, R. S. Baric, T. B. Stockwell, D. J. Spiro, M. R. Denison, Infidelity of SARS-CoV Nsp14-exonuclease mutant virus replication is revealed by complete genome sequencing. PLOS Pathog. 6, e1000896 (2010). doi:10.1371/journal.ppat.1000896 Medline

40. M. R. Denison, R. L. Graham, E. F. Donaldson, L. D. Eckerle, R. S. Baric, Coronaviruses: An RNA proofreading machine regulates replication fidelity and diversity. RNA Biol. 8, 270-279 (2011). doi:10.4161/rna.8.2.15013 Medline

41. E. C. Smith, H. Blanc, M. C. Surdel, M. Vignuzzi, M. R. Denison, Coronaviruses lacking exoribonuclease activity are susceptible to lethal mutagenesis: Evidence for proofreading and potential therapeutics. PLOS Pathog. 9, e1003565 (2013). doi:10.1371/journal.ppat.1003565 Medline

42. L. Bazak, A. Haviv, M. Barak, J. Jacob-Hirsch, P. Deng, R. Zhang, F. J. Isaacs, G. Rechavi, J. B. Li, E. Eisenberg, E. Y. Levanon, A-to-I RNA editing occurs at over a hundred million genomic sites, located in a majority of human genes. Genome Res. 24, 365-376 (2014). doi:10.1101/gr.164749.113 Medline

43. D. Bar-Yaacov, G. Avital, L. Levin, A. L. Richards, N. Hachen, B. Rebolledo Jaramillo, A. Nekrutenko, R. Zarivach, D. Mishmar, RNA-DNA differences in human mitochondria restore ancestral form of $16 S$ ribosomal RNA. Genome Res. 23, 1789-1796 (2013). doi:10.1101/gr.161265.113 Medline

44. K. A. Lehmann, B. L. Bass, Double-stranded RNA adenosine deaminases ADAR1 and ADAR2 have overlapping specificities. Biochemistry 39, 12875-12884 (2000). doi:10.1021/bi001383g Medline

45. J. B. Li, E. Y. Levanon, J.-K. Yoon, J. Aach, B. Xie, E. Leproust, K. Zhang, Y. Gao, G. M. Church, Genome-wide identification of human RNA editing sites by parallel DNA capturing and sequencing. Science 324, 1210-1213 (2009). doi:10.1126/science.1170995 Medline

46. J. H. Bahn, J.-H. Lee, G. Li, C. Greer, G. Peng, X. Xiao, Accurate identification of Ato-I RNA editing in human by transcriptome sequencing. Genome Res. 22, 142150 (2011). doi:10.1101/gr.124107.111 Medline

47. E. Picardi, C. Manzari, F. Mastropasqua, I. Aiello, A. M. D'Erchia, G. Pesole, Profiling RNA editing in human tissues: Towards the inosinome Atlas. Sci. Rep. 5, 14941 (2015). doi:10.1038/srep14941 Medline

48. E. Picardi, A. M. D’Erchia, C. Lo Giudice, G. Pesole, REDlportal: A comprehensive database of A-to-I RNA editing events in humans. Nucleic Acids Res. 45 (D1), D750-D757 (2016). doi:10.1093/nar/gkw767 Medline

49. B. R. Rosenberg, C. E. Hamilton, M. M. Mwangi, S. Dewell, F. N. Papavasiliou, Transcriptome-wide sequencing reveals numerous APOBEC1 mRNA-editing targets in transcript 3' UTRs. Nat. Struct. Mol. Biol. 18, 230-236 (2011). doi:10.1038/nsmb.1975 Medline

50. T. Lerner, F. N. Papavasiliou, R. Pecori, RNA Editors, Cofactors, and mRNA Targets: An Overview of the C-to-U RNA Editing Machinery and Its Implication in Human Disease. Genes (Basel) 10, 13 (2018). doi:10.3390/genes10010013 Medline

51. S. H. Roth, E. Y. Levanon, E. Eisenberg, Genome-wide quantification of ADAR adenosine-to-inosine RNA editing activity. Nat. Methods 16, 1131-1138 (2019). doi:10.1038/s41592-019-0610-9 Medline

52. S. Osenberg, D. Dominissini, G. Rechavi, E. Eisenberg, Widespread cleavage of Ato-I hyperediting substrates. RNA 15, 1632-1639 (2009). doi:10.1261/rna.1581809 Medline

53. N. L. Ko, E. Birlouez, S. Wain-Hobson, R. Mahieux, J.-P. Vartanian, Hyperediting of human T-cell leukemia virus type 2 and simian T-cell leukemia virus type 3 by the dsRNA adenosine deaminase ADAR-1. J. Gen. Virol. 93, 2646-2651 (2012). doi:10.1099/vir.0.045146-0 Medline

54. H. T. Porath, S. Carmi, E. Y. Levanon, A genome-wide map of hyper-edited RNA reveals numerous new sites. Nat. Commun. 5, 4726 (2014) doi:10.1038/ncomms5726 Medline

55. A. Chester, A. Somasekaram, M. Tzimina, A. Jarmuz, J. Gisbourne, R. O'Keefe, J. Scott, N. Navaratnam, The apolipoprotein B mRNA editing complex performs a multifunctional cycle and suppresses nonsense-mediated decay. EMBO J. 22 , 3971-3982 (2003). doi:10.1093/emboj/cdg369 Medline

56. S. Sharma, S. K. Patnaik, Z. Kemer, B. E. Baysal, Transient overexpression of exogenous APOBEC3A causes C-to-U RNA editing of thousands of genes. RNA Biol. 14, 603-610 (2017). doi:10.1080/15476286.2016.1184387 Medline

57. J. M. Kidd, T. L. Newman, E. Tuzun, R. Kaul, E. E. Eichler, Population stratification of a common APOBEC gene deletion polymorphism. PLOS Genet. 3, e63 (2007). doi:10.1371/journal.pgen.0030063 Medline

58. J. Long, R. J. Delahanty, G. Li, Y.-T. Gao, W. Lu, Q. Cai, Y.-B. Xiang, C. Li, B.-T. Ji, Y. Zheng, S. Ali, X.-O. Shu, W. Zheng, A common deletion in the APOBEC3 genes and breast cancer risk. J. Natl. Cancer Inst. 105, 573-579 (2013). doi:10.1093/inci/dit018 Medline

59. A. M. Bolger, M. Lohse, B. Usadel, Trimmomatic: A flexible trimmer for Illumina sequence data. Bioinformatics 30, 2114-2120 (2014). doi:10.1093/bioinformatics/btu170 Medline

60. H. Li, R. Durbin, Fast and accurate short read alignment with Burrows-Wheeler transform. Bioinformatics 25, 1754-1760 (2009). doi:10.1093/bioinformatics/btp324 Medline

61. H. Li, B. Handsaker, A. Wysoker, T. Fennell, J. Ruan, N. Homer, G. Marth, G. Abecasis, R. Durbin, 1000 Genome Project Data Processing Subgroup, The Sequence Alignment/Map format and SAMtools. Bioinformatics 25, 2078-2079 (2009). doi:10.1093/bioinformatics/btp352 Medline

62. K. Okonechnikov, A. Conesa, F. García-Alcalde, Qualimap 2: Advanced multisample quality control for high-throughput sequencing data. Bioinformatics 32 , 292-294 (2016). Medline

63. M. Lawrence, W. Huber, H. Pagès, P. Aboyoun, M. Carlson, R. Gentleman, M. T. Morgan, V. J. Carey, Software for computing and annotating genomic ranges. PLOS Comput. Biol. 9, e1003118 (2013). doi:10.1371/journal.pcbi.1003118 Medline

64. R. C. Edgar, MUSCLE: Multiple sequence alignment with high accuracy and high 
throughput. Nucleic Acids Res. 32, 1792-1797 (2004). doi:10.1093/nar/gkh340 Medline

\section{ACKNOWLEDGMENTS}

In memory of Li Wenliang, Carlo Urbani and of all the doctors and health workers who endangered their lives in the fight against epidemics. We acknowledge and thank all authors that are sharing their data. We gratefully acknowledge the authors, originating and submitting laboratories, of the sequences from GISAID's EpiFlu Database on which this research is based. The list is detailed in Data file S6. Funding: The research was supported by grants from Ministero della Salute [PE-2013-02357669] and from AIRC [IG-17701]. Author contributions: Conceptualization: SDG, FM, MGT, GM, SGC; Formal Analysis, Investigation, Software: SDG, FM; Visualization: GM; Writing - original draft: GM and SGC; Writing - review and editing: SDG, FM, MGT, GM, SGC. Competing interests: Authors declare no competing interests. Data and materials availability: Sequencing and genomic data are available through NCBI SRA and Virus repositories, and GISAID.

\section{SUPPLEMENTARY MATERIALS}

advances.sciencemag.org/cgi/content/full/sciadv.abb5813/DC1

Submitted 04 March 2020

Accepted 05 May 2020

Published First Release 18 May 2020

10.1126/sciadv.abb5813 

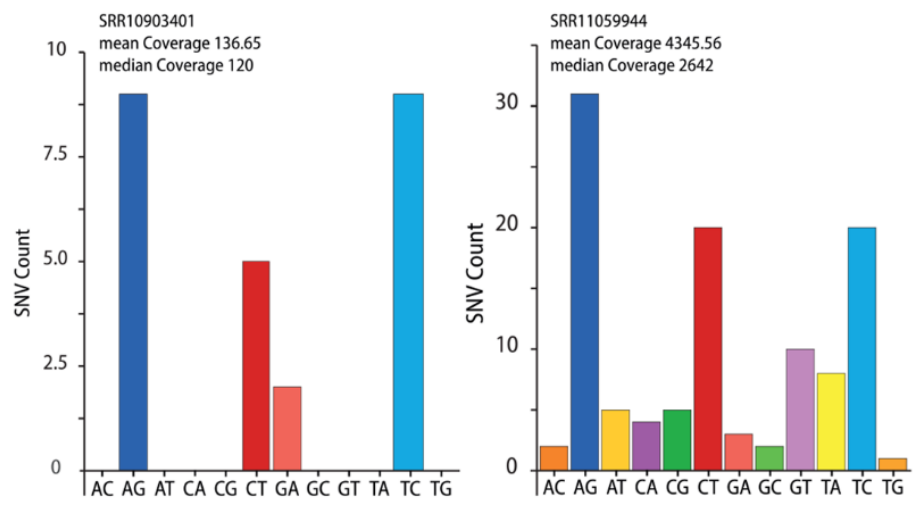

Fig. 1. Single-nucleotide variants (SNV) identified in SARS-CoV-2 transcriptomes. The bar charts show the number of SNVs identified in each SARS-CoV-2 transcriptome for each SNV type (e.g., A>C, AC). The sequencing depth for each sample is indicated.
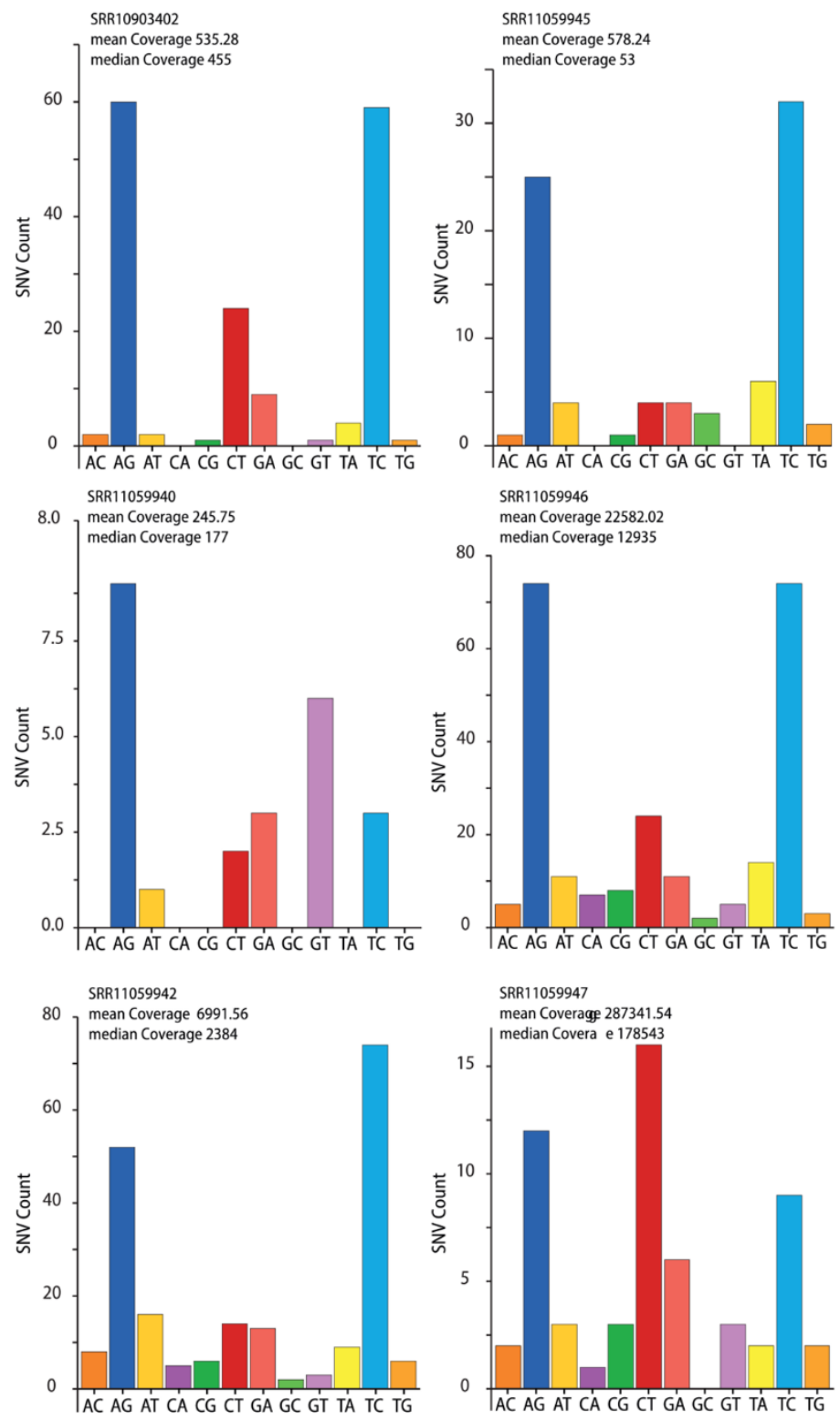

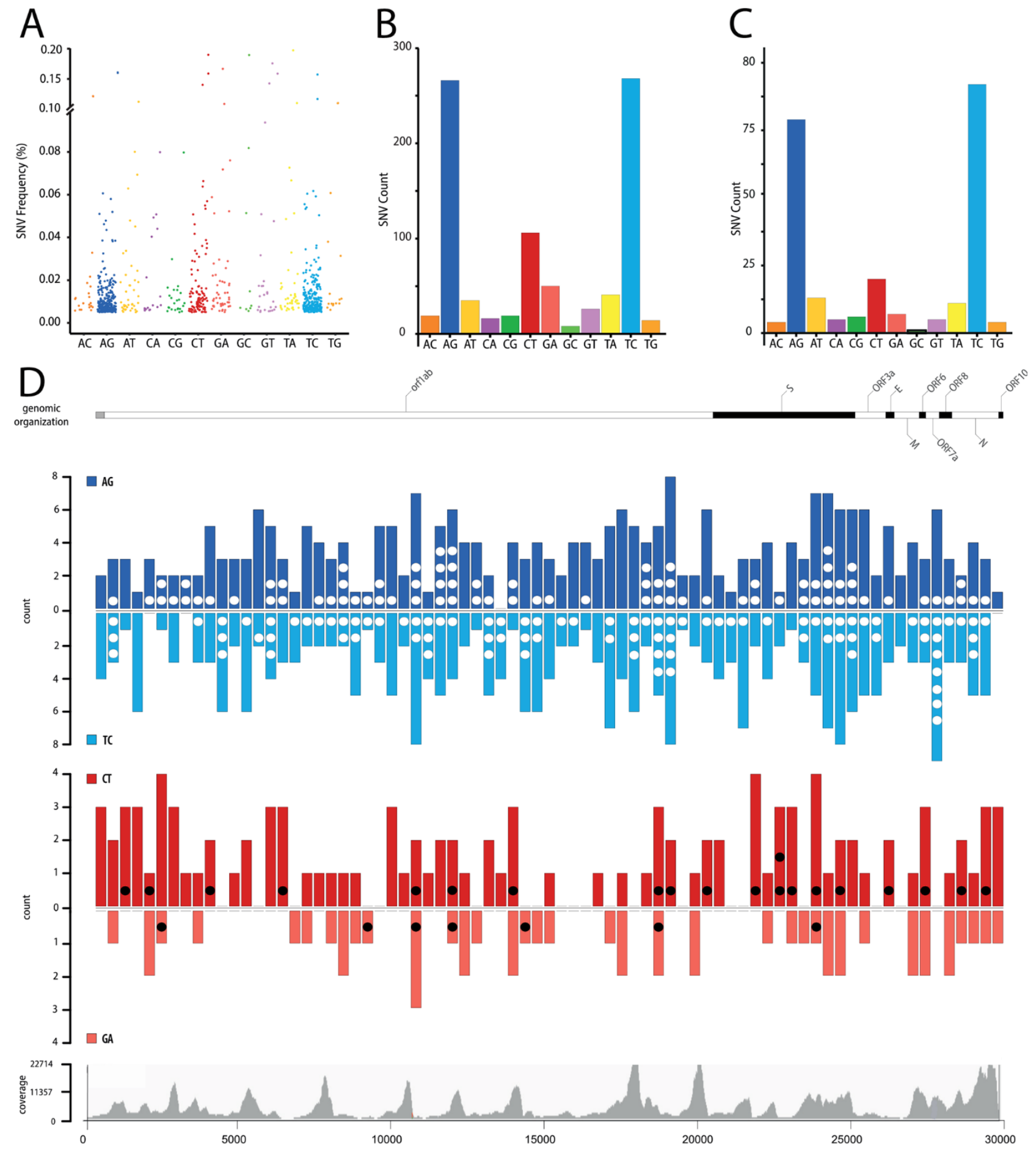

Fig. 2. Single-nucleotide variants (SNV) identified in SARS-CoV-2 transcriptomes. (A) Allelic fraction and (B) number of SNVs for each nucleotide change in the entire dataset and (C) for SNVs recurring in at least two samples. (D) Distribution of SNVs across the SARS-CoV-2 genome. A-to-G (blue) and C-to$\mathrm{U}$ (red) SNVs are grouped in 400nt bins and plotted above (AG and CT) or below the line (TC and GA) based on the edited strand. Dots (white/black) indicate recurring SNVs. Genetic organization of SARSCoV-2 (top), the dark/white shading indicates the viral coding sequences; coverage distribution of all analyzed samples (bottom). 

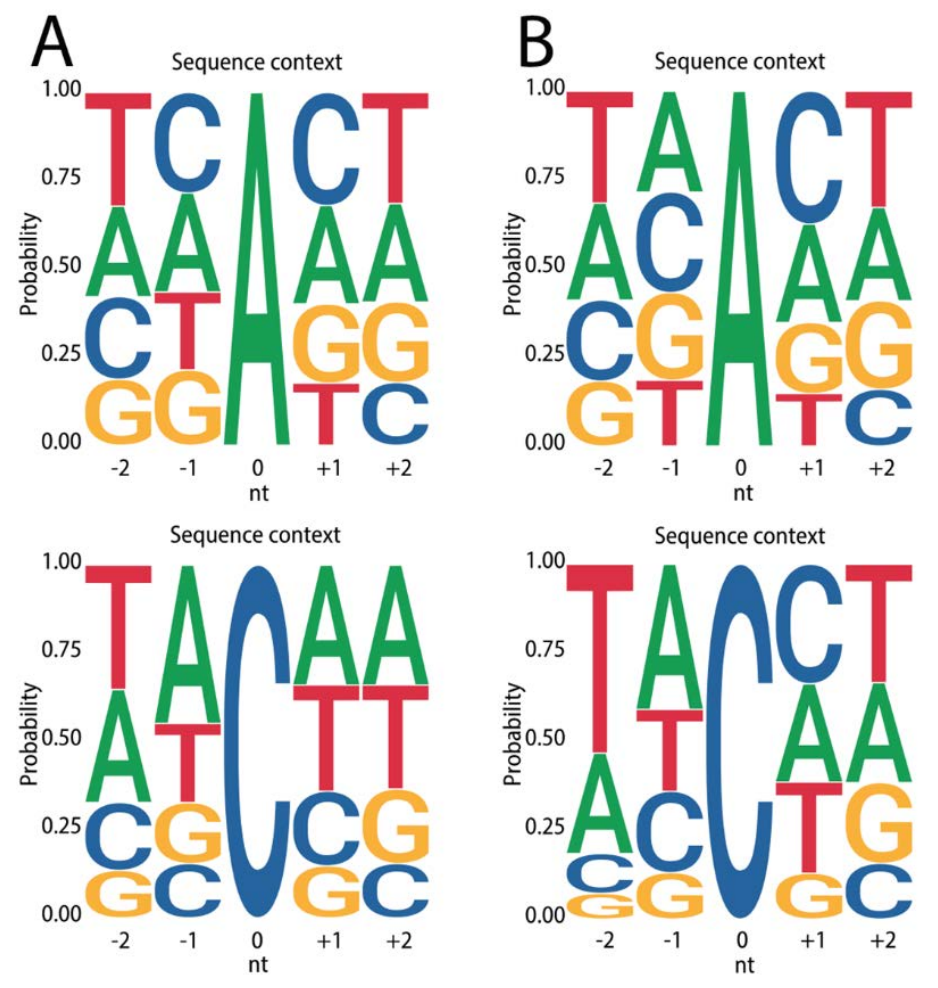

Fig. 3. Sequence contexts for SARS-CoV-2 RNA edited sites. (A) Local sequence context for A-to-I and $\mathrm{C}$-to- $\mathrm{U}$ edited sites in the viral transcriptome and (B) for recurring sites. 

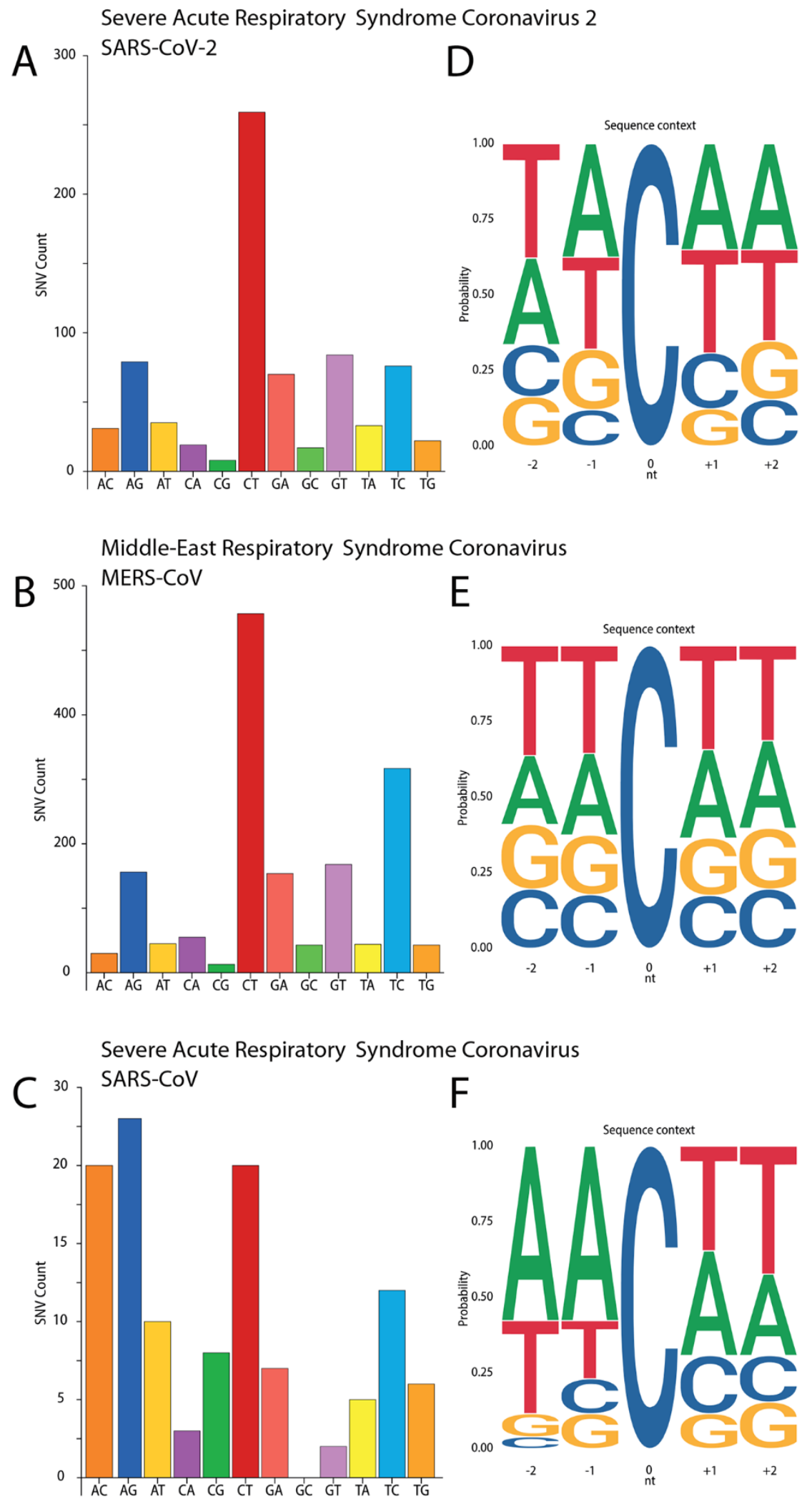

Fig. 4. Nucleotide changes across Coronaviridae strains. (A, $B, C)$ Number of SNVs for each nucleotide change and (D, E, F) local sequence context for $\mathrm{C}$-to- $U$ edited sites in genome alignments from SARS-CoV-2 (A, D), humanhosted MERS-CoV (B, E), and human-hosted SARS-CoV (C, F). 

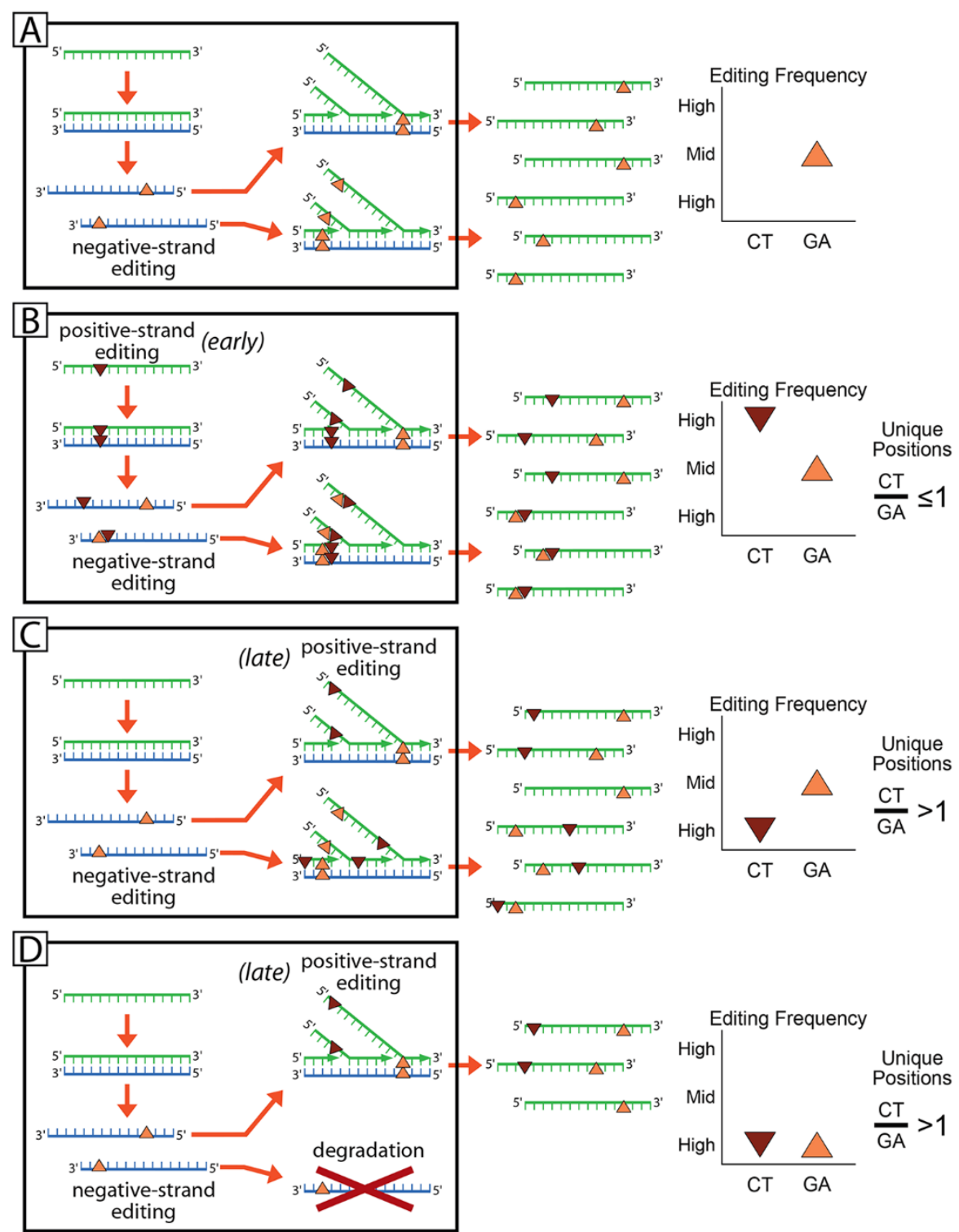

Fig. 5. Model of APOBEC RNA editing on SARS-CoV-2 transcriptome. The 4 panels model the editing frequencies and the $C>U / G / A$ ratios expected from 4 different scenarios: (A) C-to- $U$ editing on the negativesense transcripts; (B) 'early' editing on the viral genomes before viral replication; (C) 'late' editing after viral replication; (D) 'late' editing after viral replication with loss of negative-sense transcripts. Red dots indicate editing on the positive-sense transcript; Orange dots indicate editing on the positive-sense transcript. Green and Blue segments indicate positive- and negative-sense viral transcripts, respectively. 